\title{
Erratum
}

\section{Calcified Tissue International: Volume 75, Number 1, July 2004, pp 78-84 (DOI: 10.1007/s00223-003-0161-y)}

In the paper by Caballero-Alías, Loveridge, Lyon, DasGupta, Pitsillides, and J. Reeve (Calcified Tissue International, volume 75, number 1, 2004, pp. 78-84, DOI: $10.1007 / \mathrm{s} 00223-003-0161-y)$, Figure 1 should have appeared in color. Below is the color version of Fig. 1 (please note that Fig. 1 does appear in color in the online version of the article in both the html and pdf versions): 

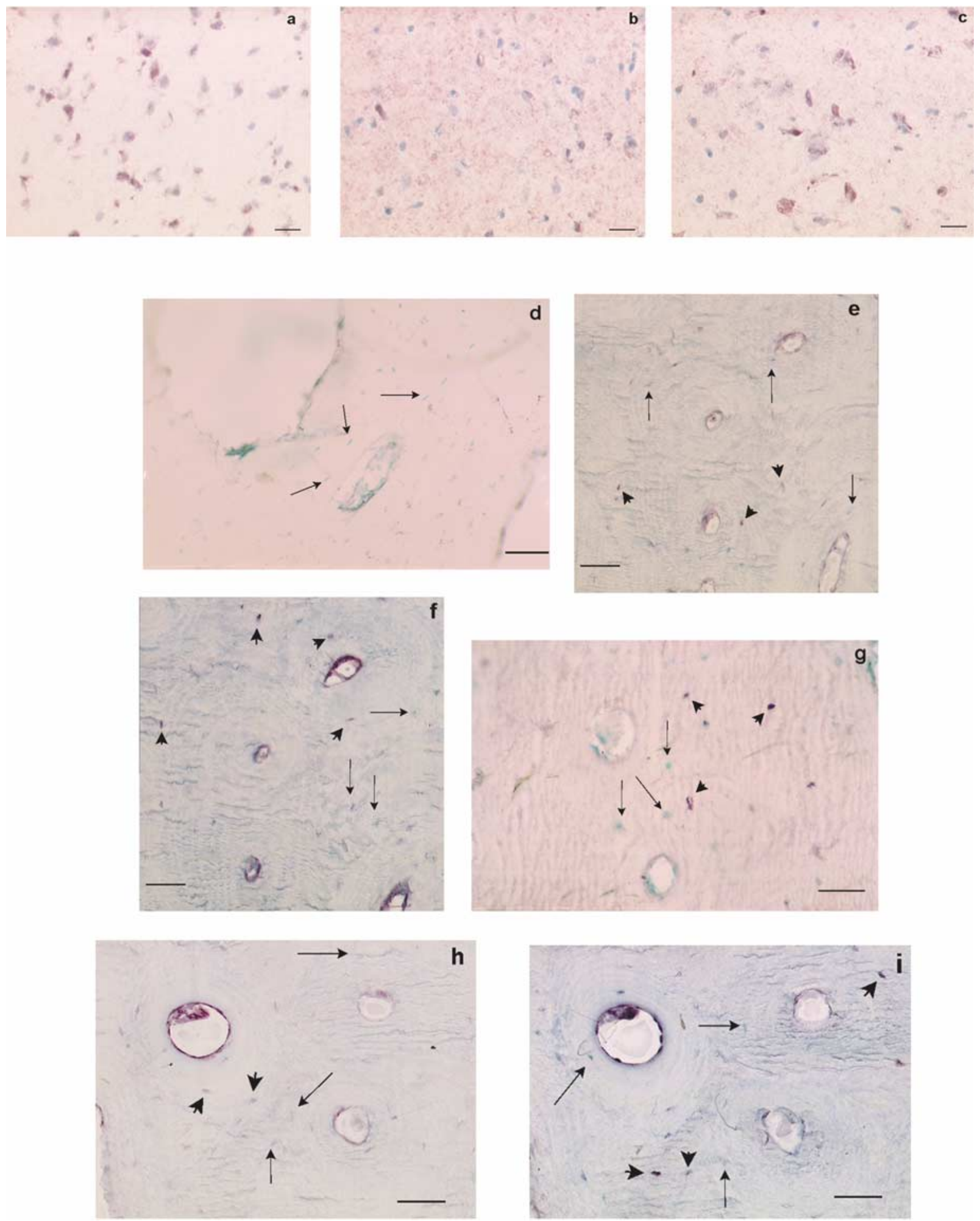

Fig. 1. (a) Rat brain section showing immunolabeling for nNOS N31020 (Transduction labs), (b) nNOS SC-648 (Santa Cruz Biotechnology) and (c) nNOS SC-1025 (Santa Cruz), (d) photomicrograph of a control for nNOS immunoreactivity in the iliac crest biopsy from the same osteoporotic patient (mouse serum was used instead of nNOS antibody), photomicrograph of serial sections for (e) nNOS and (f) eNOS immunoreactivity in the iliac crest biopsy from the same osteoporotic patient, (g) photograph of nNOS staining in the cortical bone

of a femoral neck from a non-osteoporotic postmortem biopsy, photomicrograph of serial sections for (h) eNOS and (i) nNOS immunoreactivity; sections were pre-incubated with nNOS and eNOS antisera respectively for 2 hours before overnight incubation with eNOS and nNOS. Arrowheads point to NOS positive osteocytes and arrows point to negative osteocytes counterstained with methyl green. (a), (b), and (c) bar $=20$ $\mathrm{z} \mu \mathrm{m}$. (d), (e), (f), (g), (h), and (i) bar $=50 \mu \mathrm{m}$. 